\title{
A differentially conserved residue (lle42) of GH42 $\beta$-galactosidase from Geobacillus stearothermophilus BgaB is involved in both catalysis and thermostability
}

\author{
Yi-Ning Dong, ${ }^{*} \dagger$ Hai-Qin Chen, $\${ }^{1}$ Yan-Hui Sun, ${ }^{*}$ Hao Zhang, $\ddagger$ and Wei Chen†‡ \\ ${ }^{*}$ College of Biology and Food Engineering, Chuzhou University, Chuzhou 239000, P. R. China \\ †State Key Laboratory of Food Science and Technology, School of Food Science and Technology, Jiangnan University, Wuxi 214122, P. R. China \\ ¥The Synergistic Innovation Center for Food Safety and Nutrition, Wuxi 214122, P. R. China
}

\begin{abstract}
The glycoside hydrolase family 42 (GH42) of thermophilic microorganisms consists of thermostable $\beta$-galactosidases that display significant variations in their temperature optima and stabilities. In this study, we compared the substrate binding modes of 2 GH42 $\beta$-galactosidases, BgaB from Geobacillus stearothermophilus and A4- $\beta$-Gal from Thermus thermophilus A4. The A4- $\beta-$ Gal has a catalytic triad (Glu312-Arg32Glu35) with an extended hydrogen bond network that has not been observed in BgaB. In this study, we performed site-saturation mutagenesis of Ile42 in BgaB (equivalent to Glu312 in A4- $\beta$-Gal) to study the effects of different residues on thermostability, catalytic function, and the extended hydrogen bond network. Our experimental results suggest that substitution of Ile42 with polar AA enhanced the thermostability but decreased the catalytic efficiency of BgaB. Polar AA substitution for Ile42 simultaneously affected thermostability, catalytic efficiency, and the hydrogen bond network, suggesting that Ile42 is responsible for functional discrimination between members of the GH42 family. These observations could lead to a novel strategy for investigating the functional evolution of the GH42 $\beta$-galactosidases.
\end{abstract}

Key words: $\beta$-galactosidase, glycoside hydrolase family GH42, thermostability, saturation mutagenesis, differentially conserved residue

\section{INTRODUCTION}

$\beta$-Galactosidase ( $\beta$-D-galactoside galactohydrolase; EC 3.2.1.23) exhibits both hydrolytic and transgalactosylation activities toward the $\beta(1 \rightarrow 4)$ linkage of $\beta$-Dgalactopyranosides (Torres et al., 2010). Its hydrolytic activity has been mainly applied in the dairy industry

Received November 16, 2014.

Accepted December 19, 2014

${ }^{1}$ Correspondence author: haiqinchen@jiangnan.edu.cn to improve the digestibility, solubility, and sweetness of lactose, and to produce lactose-free milk products for lactose-intolerant people. Its transgalactosylation potential has been utilized to produce prebiotic galacto-oligosaccharides and to improve the structural and functional properties of food materials or pharmaceutical compounds (Duarte et al., 2010; Gupta et al., 2012).

The $\beta$-galactosidases constitute a large family of proteins that have been found in 5 principal carbohydrateactive enzyme families (CAZy, http://www.cazy.org/) to date; namely, glycoside hydrolase (GH)1, GH2, GH35, GH42, and GH59, all of which belong to clan GH-A. Most $\beta$-galactosidases from thermophiles, psychrophiles, and halophiles belong to GH42. Enzymes in GH42 are believed to use a 2 -step, double-displacement mechanism in which a covalent glycosyl-enzyme intermediate is formed and hydrolyzed via oxocarbenium ion-like transition states (Viborg et al., 2014). Thermostable $\beta$-galactosidases produced by moderate thermophiles and hyperthermophiles offer several biotechnological applications and advantages, such as greater stability, higher solubility of lactose in the aqueous phase, higher product yield, increased reaction rates, tolerance to pasteurization, and elimination of microbial contamination (Petzelbauer et al., 1999; Ji et al., 2005). In addition to the growing market for functional food production, industrial interest in thermostable $\beta$-galactosidases (cold-adapted or thermophilic) will likely increase. Thermostable $\beta$-galactosidase exhibits relatively poor yield and catalytic activity when purified from the original host, although the enzyme is well suited for industrial use because of its high thermostability (Chen et al., 2008; Park et al., 2010; Marin-Navarro et al., 2014). However, recombinant microbial expression systems have improved production and biotechnological applicability of $\beta$-galactosidase (Schmidt and Stougaard, 2010; Oliveira et al., 2011). Overexpression of thermophilic enzymes in Escherichia coli or Saccharomyces cerevisiae allows high enzyme yields and relatively easy purification by heat treatment. However, the activity of $\beta$-galactosidase has been 
shown to decrease through loss of its thermostability during its expression and purification (Xia et al., 2010). Current developments in thermostable $\beta$-galactosidase, derived from newly identified microbial sources or by protein engineering means, together with the use of efficient recombinant microbial systems, are transforming this enzyme into a relevant synthetic tool. However, the reasons for the decrease in enzyme activity remain unclear.

In the GH42 $\beta$-galactosidase A4- $\beta$-Gal from Thermus thermophilus A4, investigation of the microenvironment of substrate-binding residues has revealed that the nucleophile Glu312 does not work independently but requires assistance from an interaction network involving 2 other residues (Arg32 and Asn140). Although variability in residue Arg32 has been observed, similar interaction networks have been found in $\beta$-galactosidase BgaB from Geobacillus stearothermophilus and 2 other members of GH42: Bca- $\beta$-Gal and BgaP. To investigate the function of the equivalent residue in $\mathrm{BgaB}$, we constructed and characterized Ile42 variants to identify the microenvironments responsible for some of the differences in thermal stability and activity between the homologous enzymes. Structural and biochemical studies of these variants have provided insight into how differentially conserved residues contribute to $\beta$-galactosidase BgaB thermal stability and catalytic activity. This knowledge can serve as a structural toolbox for the design of $\beta$-galactosidase with specific properties and features suited to existing or new applications.

\section{MATERIALS AND METHODS}

\section{Strain, Plasmids, and Culture Conditions}

The host strain used was E. coli BL21 (DE3) $\left[F^{-}\right.$, omp T, hsdSB $\left(r B^{-} m B^{-}\right)$, gal ( $\lambda$ c I 85\%, ind1, Sam\%, nin5, lacUV5-T\%gene1), and dcm (DE3)] from Novagen (Madison, WI). Plasmid pET28a (+), obtained from the Culture Collection of Food Microorganisms of Jiangnan University (CCFM, Jiangnan, China) was used as the expression vector for BgaB. Recombinant $E$. coli strains were routinely grown at $37^{\circ} \mathrm{C}$ with orbital shaking at $200 \mathrm{rpm}$ in Luria Bertani medium alone or supplement- ed with $50 \mu \mathrm{g} / \mathrm{mL}$ kanamycin (LBK). Sequencing of DNA and oligonucleotide synthesis were performed by Shanghai Biosune Biotechnology Co. Ltd. (Shanghai, China). All other chemicals were analytical reagent grade and purchased from Shanghai Sangon Biological Engineering Technology \& Services (Shanghai, China).

\section{Site-Saturation Mutagenesis}

Site-saturation mutants I42X of BgaB were constructed following the site overlap extension PCR method (Arnold and Georgiou, 2003); XXX (Table 1) indicates the respective codons that were mutated directly on pET28a- $b g a B$. This strategy used 2 sequential PCR reactions, using 2 flanking primers (the forward and reverse primers that were used for cloning the $b g a B$ gene) and 2 different internal primers designed to introduce the mutations. The reverse primer together with the forward mutagenic internal primer and the forward primer together with the internal reverse primer were used to amplify 2 overlapping fragments that were subsequently annealed. The forward and reverse flanking primers were then used to amplify the annealed mutated $b g a B$ gene. Instead of using degenerate primers, 19 sense primers bearing respective mutation codons and a universal antisense primer were synthesized, and the PCR was run separately 19 times. The flanking and internal primers are shown in Table1. The mutated nucleotides are shown in bold and the restriction sites for $N c o I$ and $X h o I$ are underlined. After enzymatic characterization of each mutant, all mutant DNAs were recovered from $E$. coli cells and the mutation positions were confirmed by DNA sequencing (Table 1).

\section{Protein Expression and Purification}

After overnight growth at $37^{\circ} \mathrm{C}$ on LBK agar plates, colonies were picked with sterile toothpicks and grown in LBK liquid medium. Isopropyl $\beta$-D-1thiogalactopyranoside was added to the culture at a final concentration of $1 \mathrm{mM}$. When the cell density reached an optical density (at $600 \mathrm{~nm}$ ) of 0.6 to 0.8 , the cultures were grown further at $30^{\circ} \mathrm{C}$. After a 20 -h induction, the cell pellet was harvested by centrifugation at $8,000 \times g$ for $5 \mathrm{~min}$ at $4^{\circ} \mathrm{C}$, washed twice with $50 \mathrm{mM}$

Table 1. Nucleotide sequences of primers used in site-saturation mutagenesis ${ }^{1}$

\begin{tabular}{|c|c|}
\hline Primer & Sequence \\
\hline N-terminal primers & 5'-GGGCCCCCATGGGCATGAATGTGTTATCCTCAAT-3' \\
\hline C-terminal primers & 5'-CCGCTCGAGAACCTTCCCGGCTTCATCAT-3' \\
\hline Internal primer: I42X & 5'-TCTTTAGGGXXXTTCAGTTG-3' \\
\hline
\end{tabular}


potassium phosphate buffer $\mathrm{pH}$ 6.5, and then disrupted by sonication on ice for $10 \mathrm{~min}$. Cell debris was removed by centrifugation at $12,000 \times g$ for $10 \mathrm{~min}$ at $4^{\circ} \mathrm{C}$. Recombinant His-tagged enzymes were purified using a Ni-chelating Sepharose Fast Flow column (Qiagen, Shanghai, China) based on a previous report (Dong et al., 2011). Homogeneous fractions were pooled and imidazole was removed using an Amicon Ultra Centrifugal Filter Device (EMD Millipore, Billerica, MA) with a $30-\mathrm{kDa}$ cut-off membrane. Protein preparations were evaluated by SDS-PAGE and Coomassie Blue staining. All purification steps were carried out $4^{\circ} \mathrm{C}$.

\section{Characterization of BgaB and its Mutants}

Substrate-free enzymes were used for measuring specific activities at the optimal temperatures and pH as previously described (Dong et al., 2011). Protein concentrations were determined by the method of Bradford (1976) with BSA as standard. The enzymes were assayed in a temperature range of 20 to $70^{\circ} \mathrm{C}$, and the optimum $\mathrm{pH}$ test was performed with $50 \mathrm{mM}$ potassium phosphate adjusted to the appropriate $\mathrm{pH}$ values in the range of 5.0 to 9.0 with either $3 \mathrm{M} \mathrm{KOH}$ or $3 \mathrm{M} \mathrm{HCl}$.

For the $\mathrm{pH}$ stability assay, samples were incubated at $\mathrm{pH} 6.5$ and $55^{\circ} \mathrm{C}$ and then transferred to ice at different time points. Residual enzyme activity was assayed with ortho-nitrophenyl- $\beta$-galactoside ( $\boldsymbol{o} \mathbf{N P G}$ ) as the substrate, as previously described (Dong et al., 2011).

\section{Thermal Inactivation Kinetics and Differential Scanning Calorimetry}

Thermal inactivation of wild-type BgaB and the mutants were determined after incubating the enzymes $(0.5 \mathrm{mg} / \mathrm{mL})$ in $50 \mathrm{~m} M$ phosphate buffer $(\mathrm{pH} 6.5)$ at $70^{\circ} \mathrm{C}$ for the specified intervals. The samples were subsequently transferred to ice and the residual activities were assayed using $o \mathrm{NPG}$ as described above. Thermal stability (half-life) studies were similarly performed, except that samples were transferred to ice at 5 -min intervals. Half-life $\left(\mathbf{T}_{\mathbf{1 / 2}}\right)$ was determined for each mutant enzyme as follows: $\ln \left(\mathrm{U}_{\mathrm{t}} / \mathrm{U}_{0}\right)=-k \mathrm{t}, \mathrm{T}_{1 / 2}=\ln 2 / k$, where $\mathrm{U}_{\mathrm{t}}, \mathrm{U}_{0}$, and $k$ are enzyme activity at $\mathrm{t}$ min, initial enzyme activity, and the apparent rate constant, respectively.

To determine the melting temperature $\left(\boldsymbol{T}_{\mathrm{m}}\right)$ of the enzymes, differential scanning calorimetry (DSC) measurements were conducted using a Perkin Elmer Pyris 1 DSC (Waltham, MA). Samples were dialyzed overnight against $50 \mathrm{mM}$ phosphate buffer ( $\mathrm{pH} 6.5)$, centrifuged at $8,000 \times g$ for $10 \mathrm{~min}$ to remove aggregates, and degassed before loading in the calorimeter. The scan- ning rate was $1^{\circ}$ per min and a protein concentration of approximately $1 \mathrm{mg} / \mathrm{mL}$ was used. Denaturation curves were determined with Microcal Origin 5.0 software (OriginLab Corp., Northampton, MA).

\section{Determination of Enzyme Kinetics Constant}

Enzyme kinetics were determined at the optimal temperature and $\mathrm{pH}$. The Michaelis constant $\left(\boldsymbol{K}_{m}\right)$ values were calculated from Lineweaver-Burk plots. A 1-min assay was used to measure the $K_{m}$ and maximum rate $\left(\boldsymbol{V}_{\max }\right)$ values of BgaB at several different concentrations of $o$ NPG. The inhibition constant $\left(K_{i}\right)$ values were determined by fitting to Cornish-Bowden plots using various concentrations of the inhibitor galactose (from 0 to $20 \mathrm{mM}$ ) with $o \mathrm{NPG}$ as the substrate (Dong, et al., 2011).

\section{Analysis for Substrate Bound to BgaB}

The 3-dimensional (3D) X-ray crystal structure of A4- $\beta$-Gal (PDB ID: 1KWG; http://www.rcsb.org/ $\mathrm{pdb}$ /explore/explore.do?structureId=1KWG) was used as a template to construct the $\beta$-galactose BgaB 3D model. The program Modeller (version 9v4; Sali et al., 1995), which enables an automated approach to comparative modeling by satisfaction of spatial restraints, was performed using standard parameters to build the 3D structure of BgaB (Bhattacharya et al., 2008; Daga et al., 2010). To understand the mechanisms of ligand binding and other interactions between the substrate and $\beta$-galactosidase, a $3 \mathrm{D}$ model of $\mathrm{BgaB}$ complexed with galactose was produced, using PatchDock (Schneidman-Duhovny et al., 2005; Mashiach et al., 2010). The program Ligplot (Wallace et al., 1995) was used to study the enzyme-substrate interactions. Structural investigations were performed using the $3 \mathrm{D}$ visualization program CCP4MG (McNicholas et al., 2011).

\section{RESULTS}

\section{Structure Comparisons and Selection of Mutation Sites}

Geobacillus stearothermophilus $\beta$-galactosidase BgaB and $T$. thermophilus A4 $\beta$-galactosidase A4- $\beta$-Gal are both thermostable members of GH42. However, their specific activities and thermal stabilities are very different from each other (Hidaka et al., 2002; Chen et al., 2008). An examination of the crystal structure of A4- $\beta$-Gal, which is the first known structure of a GH42 enzyme, revealed that this enzyme possesses a common $(\beta / \alpha)_{8}$-barrel (TIM barrel) structure and exhibits very strict recognition of the galactosyl moiety. Although 
the structure of BgaB has not been elucidated, we previously constructed a $3 \mathrm{D}$ structure of BgaB based on homology modeling using A4- $\beta$-Gal as the template (Dong et al., 2011). We predicted the substrate-binding mode of BgaB by comparative analysis with A4- $\beta$-Gal (Figure 1), and found that the nucleophilic Glu312 of A4- $\beta$-Gal does not work independently but requires assistance from an interaction network involving 2 other residues (Arg32 and Glu35) via $\mathrm{H}_{2} \mathrm{O}$ to achieve efficient catalysis. However, this catalytic triad is absent in $\mathrm{BgaB}$, as residue Ile42 is a homologous substitute for Glu35. The nonpolar side chain of Ile42 cannot form hydrogen bonds with Ser39 or nucleophilic Glu303 via $\mathrm{H}_{2} \mathrm{O}$. Thus, we hypothesized that residue Ile42 is a homologous, functionally discriminating position in BgaB. To investigate the function of Ile42 and the effect of mutating it on the catalytic triad and hydrogen bond network formation, this residue was chosen for site-saturation mutagenesis.

\section{Analysis of Activities and Stabilities of Purified Ile42 Mutants}

To investigate the functions of Ile 42 and the inferred hydrogen bonds on the activity of $\beta$-galactosidase BgaB, substitutions of Ile42 with 19 other AA were evaluated.
The mutants could be classified into polar and nonpolar AA substitutions. Based on the analysis discussed above, polar AA substitutions mutants would be able to maintain the hydrogen bond with Ser39, whereas nonpolar AA substitutions mutants would eliminate this inferred hydrogen bond of the wild-type enzyme. Table 2 gives detailed results of the enzymological characterization of the mutants. Substitution of Ile42 with Lys, Arg, His, Asp, or Glu drastically reduced the specific activity, whereas the optimal $\mathrm{pH}, \mathrm{pH}$ stability, and optimal temperature of all the mutants were not significantly changed (data not shown). As illustrated in Table 2, the Arg, Lys, Asp, His, and Glu mutants retained $39,46,62,66$, and $67 \%$, respectively, of the wild-type specific activity. However, the replacement of the Ile42 with neutral AA and other nonpolar AA did not result in an obvious loss of specific activity. The dramatic loss of activity when Ile42 was mutated to a charged AA (Glu, Asp, Lys, Arg, or His) strongly suggests that Ile42 is important for maintaining BgaB catalytic activity.

Quantitative evaluation of the thermodynamic parameters of the purified wild-type and mutant enzymes are shown in Table 2. Our results indicated that substitution of Ile42 with polar AA increased thermostability relative to the wild-type enzyme and other nonpolar
A

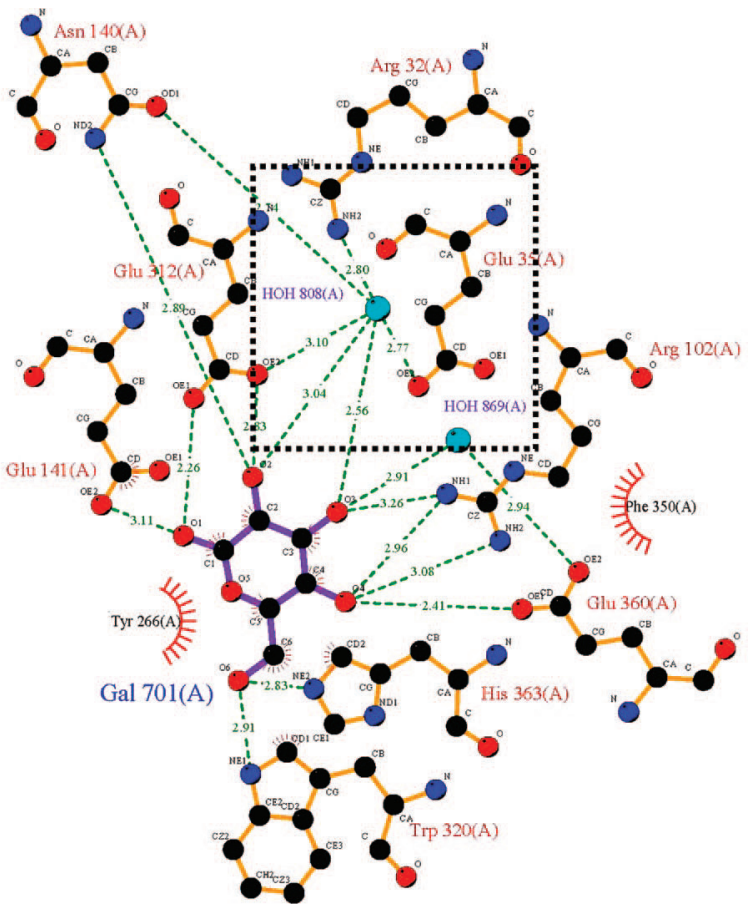

B

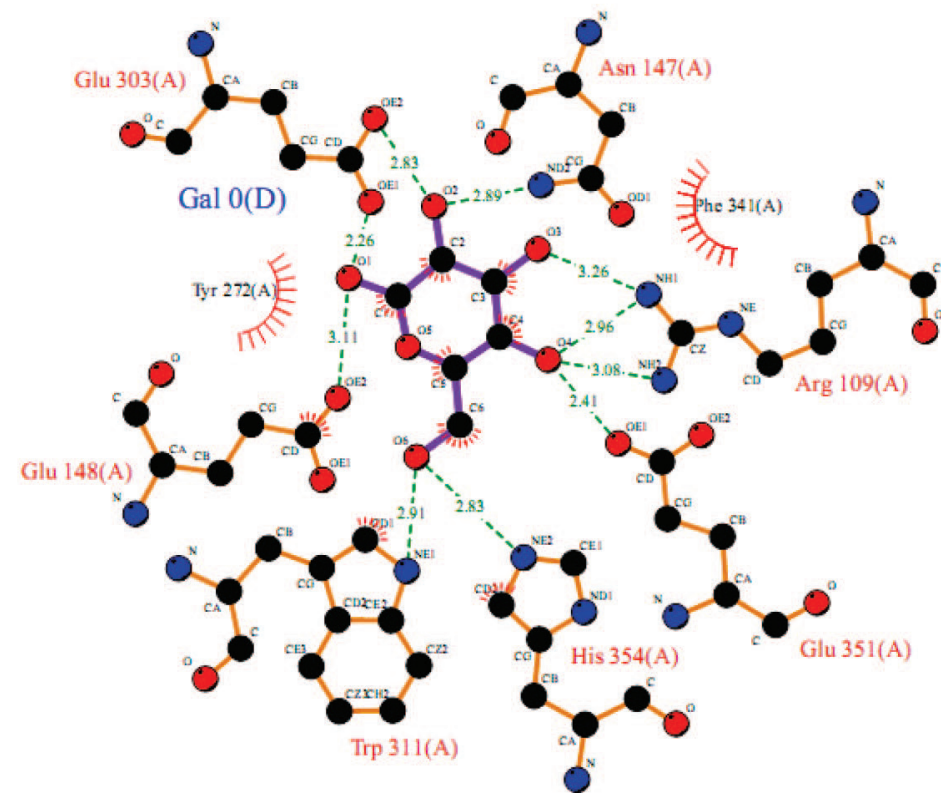

Figure 1. Schematic representation of the catalytic triads and surrounding AA residues in $2 \beta$-galactosidases - BgaB from Geobacillus stearothermophilus and A4- $\beta$-Gal from Thermus thermophilus A4 - interacting with galactose. Hydrogen bonds are shown as dotted (green) lines. (A) Hydrogen bonds of A4- $\beta$-Gal. Water is shown as separate, light-gray (light-blue) circles. (B) Hydrogen bonds of BgaB. The dotted frame indicates the difference in the polar AA position between A4- $\beta$-Gal and BgaB. Color version available online. 
Table 2. Comparative biochemical characteristics of mutated enzymes at position Ile $42^{1}$

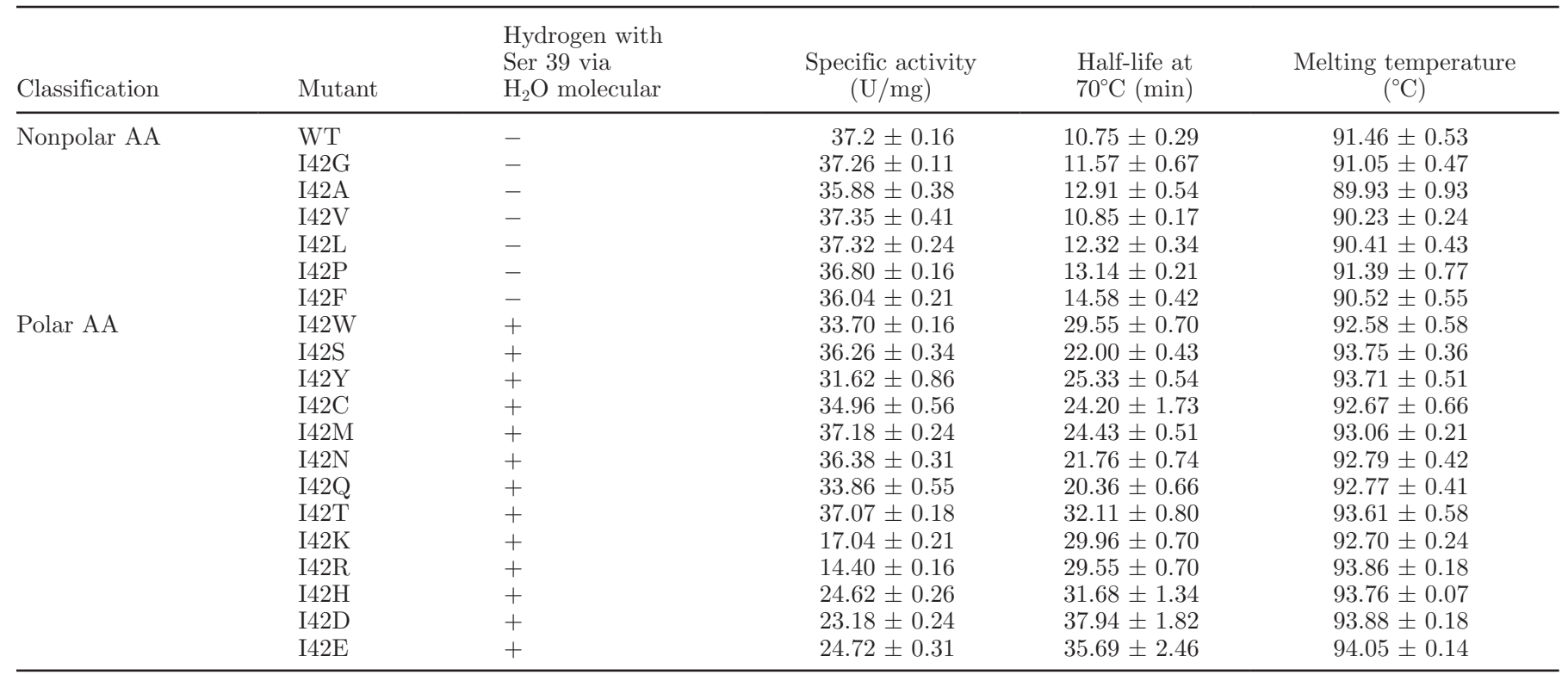

${ }^{1}$ Results are the mean \pm SE from 6 measurements.

AA substitution mutants. The $\mathrm{T}_{1 / 2}$ at $70^{\circ} \mathrm{C}$ of the wild-type enzyme and the Thr, Glu, and Asp mutants was $10.75,32.11,35.69$, and $37.94 \mathrm{~min}$, respectively. In contrast, the replacement of Ile 42 by any nonpolar AA did not significantly affect $T_{1 / 2}$. It appears that the substitution of a nonpolar AA with a polar AA plays an important role in enhancement of the enzyme thermostability. The results of the DSC experiments were consistent with these results. The Asp and Glu mutants unfolded at $93.88^{\circ} \mathrm{C}$ and $94.05^{\circ} \mathrm{C}$, and these $T_{\mathrm{m}}$ were comparable to that observed for the wild-type enzyme $\left(91.46^{\circ} \mathrm{C}\right)$. Therefore, substitutions of Ile 42 with polar AA drastically affect the catalytic activity and thermal stability of BgaB.

\section{Analysis of Steady-State Kinetics}

Kinetic parameters of wild-type and mutant BgaB were obtained and interpreted according to the substrate inhibition model. Analysis of kinetic parameters obtained for the Ile 42 mutants revealed that the turnover rates of the polar AA substitution mutants, as reflected in their turnover rate $\left(\boldsymbol{k}_{\text {cat }}\right)$ values, were approximately 11 to $63 \%$ of that of wild-type enzyme (Table 3). However, the $k_{\text {cat }}$ values for the nonpolar AA substitution mutants were similar to that of the wildtype enzyme. Polar AA substitution mutants had lower $K_{m}$ values than the wild-type enzymes. For example, the $K_{m}$ for the Thr mutant was intermediate between that of Glu and Lys mutants, and lower than the $K_{m}$ value of wild-type enzyme. As reflected by the changes in $K_{m}$ values, the substitution of Ile42 with polar AA seemed to affect substrate affinity. The decrease in the $k_{c a t}$ and $K_{m}$ values resulted in a reduction in overall catalytic efficiency of the polar AA substitution mutants. These results are consistent with the specific activities observed for these mutants.

The $K_{i}$ values for galactose with the wild-type and mutants at position 42 were obtained from the intersection points in the Cornish-Bowden plots. These results suggested that mutations at position 42 have a relevant effect on inhibition by galactose. The $K_{i}$ values of the other nonpolar AA substitutions mutants were approximately 100 to $184 \%$ of that of the wild-type enzyme. However, the $K_{i}$ values of the polar AA substitution mutants were approximately 32 to $100 \%$ of that of the wild-type enzyme. The Asp mutant showed the lowest $K_{i}$ value among these mutants. These results are consistent with the $K_{m}$ values observed for these mutants. Thus, mutation of Ile42 had an important effect on the substrate-binding mode of $\mathrm{BgaB}$ and its catalytic activity.

\section{DISCUSSION}

\section{Comparison of Substrate Recognition Between $B g a B$ and $A 4-\beta-G a l$}

Several attempts have been made previously to identify potentially important AA residues in GH42 enzymes through site-directed mutagenesis of conserved residues. The AA residues in question include those 
Table 3. Enzyme kinetics ${ }^{1}$ for the thermostable mutants (mean $\pm \mathrm{SD}$ )

\begin{tabular}{|c|c|c|c|c|}
\hline Mutant & $K_{m}(\mathrm{~m} M)$ & $k_{c a t}\left(\mathrm{~s}^{-1}\right)$ & $\begin{array}{c}\text { Relative } \\
k_{c a t} / K_{m}(\%)\end{array}$ & $K_{i}(\mathrm{~m} M)$ \\
\hline $\mathrm{WT}$ & $2.9 \pm 0.01$ & $7.56 \pm 0.05$ & 100 & $87.27 \pm 0.86$ \\
\hline I42G & $2.9 \pm 0.08$ & $7.6 \pm 0.2$ & 100 & $141.23 \pm 4.05$ \\
\hline $\mathrm{I} 42 \mathrm{~A}$ & $3.33 \pm 0.37$ & $8.37 \pm 0.93$ & 96 & $159.78 \pm 1.85$ \\
\hline $\mathrm{I} 42 \mathrm{~V}$ & $2.16 \pm 0.06$ & $5.57 \pm 0.08$ & 99 & $103.54 \pm 1.11$ \\
\hline I42L & $2.67 \pm 0.09$ & $6.99 \pm 0.23$ & 100 & $128.54 \pm 0.9$ \\
\hline $\mathrm{I} 42 \mathrm{P}$ & $2.55 \pm 0.11$ & $6.61 \pm 0.29$ & 99 & $122.57 \pm 0.89$ \\
\hline $\mathrm{I} 42 \mathrm{~F}$ & $2.96 \pm 0.06$ & $7.51 \pm 0.15$ & 97 & $142.59 \pm 1.04$ \\
\hline I42W & $1.8 \pm 0.14$ & $4.3 \pm 0.34$ & 92 & $86.69 \pm 1.07$ \\
\hline I42S & $1.26 \pm 0.07$ & $3.19 \pm 0.18$ & 97 & $61.09 \pm 1.67$ \\
\hline $\mathrm{I} 42 \mathrm{Y}$ & $1.18 \pm 0.09$ & $2.64 \pm 0.2$ & 86 & $56.39 \pm 0.93$ \\
\hline $\mathrm{I} 42 \mathrm{C}$ & $1.84 \pm 0.05$ & $4.75 \pm 0.41$ & 94 & $85.82 \pm 2.57$ \\
\hline $\mathrm{I} 42 \mathrm{M}$ & $1.49 \pm 0.08$ & $3.81 \pm 0.33$ & 98 & $71.58 \pm 0.96$ \\
\hline $\mathrm{I} 42 \mathrm{~N}$ & $1.48 \pm 0.14$ & $3.81 \pm 0.35$ & 99 & $71.46 \pm 1.28$ \\
\hline $\mathrm{I} 42 \mathrm{Q}$ & $1.6 \pm 0.08$ & $3.82 \pm 0.18$ & 91 & $76.68 \pm 0.69$ \\
\hline $\mathrm{I} 42 \mathrm{~T}$ & $1.55 \pm 0.1$ & $3.76 \pm 0.31$ & 93 & $74.6 \pm 1.2$ \\
\hline $\mathrm{I} 42 \mathrm{~K}$ & $1.06 \pm 0.12$ & $1.27 \pm 0.15$ & 46 & $50.84 \pm 0.97$ \\
\hline I42R & $0.8 \pm 0.17$ & $0.81 \pm 0.18$ & 39 & $38.29 \pm 0.9$ \\
\hline $\mathrm{I} 42 \mathrm{H}$ & $0.93 \pm 0.19$ & $1.61 \pm 0.33$ & 66 & $44.54 \pm 0.53$ \\
\hline I42D & $0.59 \pm 0.03$ & $0.96 \pm 0.05$ & 62 & $28.45 \pm 0.86$ \\
\hline $\mathrm{I} 42 \mathrm{E}$ & $0.72 \pm 0.07$ & $1.25 \pm 0.12$ & 67 & $35.76 \pm 1.19$ \\
\hline
\end{tabular}

involved in binding of the substrate in the TIM-barrel domain (Hidaka et al., 2002), evolutionarily relevant residues (Placier et al., 2009), and residues adjacent to the substrate-binding domain (Park et al., 2010). Identification of other catalytic residues was based largely on structural analyses. It has been reported that in the X-ray crystal structure of the galactosecomplex structure of A4- $\beta$-Gal complex, 1 galactose molecule is bound in the chair conformation and its $\mathrm{O} 1$ is in the $\alpha$-anomeric configuration (Hidaka et al., 2002). Figure 1A shows the bound galactose molecule and a schematic representation of the surrounding AA residues interacting with it. There are 11 direct hydrogen bonds between protein atoms and the $\mathrm{OH}$ groups of galactose in the galactose-A4- $\beta$-Gal complex. In addition, several water-mediated hydrogen bonds are present. For example, Glu35 is an indirect participant in substrate recognition by A4- $\beta$-Gal. The side chain of Glu35 recognizes O2 of galactose via a water molecule shared with Arg32. Based on the structure alignment, Ser39 and Ile42 are located in the equivalent positions in BgaB. As the absence of the polar side chain of residue Ile42, the hydrogen bond between Ser39 and Ile42 was absent in BgaB (Figure 1B). Notably, all 5 $\mathrm{OH}$ groups of galactose are hydrogen bonded with 2 or more residues, and recognition of the galactosyl moiety is very strict in both $\mathrm{BgaB}$ and $\mathrm{A} 4-\beta$-Gal (Figure 1). Hydrogen bonds between the enzyme and substrate are crucial for the hydrolysis reaction (Desai et al., 2014; Hangasky et al., 2014; Tiwari et al., 2014). Therefore, the Ile42 residue may play a key role in the formation of hydrogen bonds between the enzyme and $\mathrm{O} 2$ of ga- lactose. Thus, the absence of these hydrogen bonds in BgaB plausibly accounts for the observed differences in substrate recognition and catalytic activity from A4- $\beta$ Gal. Our results show that substitution of residue Ile42 with polar AA markedly increased substrate affinity relative to the wild-type enzyme and nonpolar AA substitution mutants, as reflected by the $K_{i}$ and $K_{m}$ values (Table 3), which are consistent with this hypothesis.

\section{Structural Localization of Ile42}

We propose that residue Ile42 assists the catalytic base nucleophile (Glu303) of BgaB. Mutations of Ile42 to either positively charged (Lys, Arg, and His) or negatively charged (Asp and Glu) residues led to a decrease in specific activity and an increase in thermal stability, respectively (Table 2). Thus, our data show a different effect on catalytic activity than the previous report, which showed that the presence of a positive charge near the catalytic nucleophile glutamate resulted in an increase in $k_{\text {cat }}$ (Lacombe-Harvey et al., 2013).

Enzyme activity and stability are influenced by multiple factors (Daudé et al., 2013; Ghollasi et al., 2013; Surzhik et al., 2014), including hydrogen bonding networks, hydrophobic interactions, entropic effects, packing efficiency, multimerization, and AA composition (McLachlan et al., 2008). Furthermore, our data suggest that Ile42 could have another function in the enzyme mechanism. Based on our molecular model, Ser39 and Ile42 residues are located at the periphery of the BgaB structure (Figure 2). The homology model showed that BgaB possesses a TIM barrel domain, 


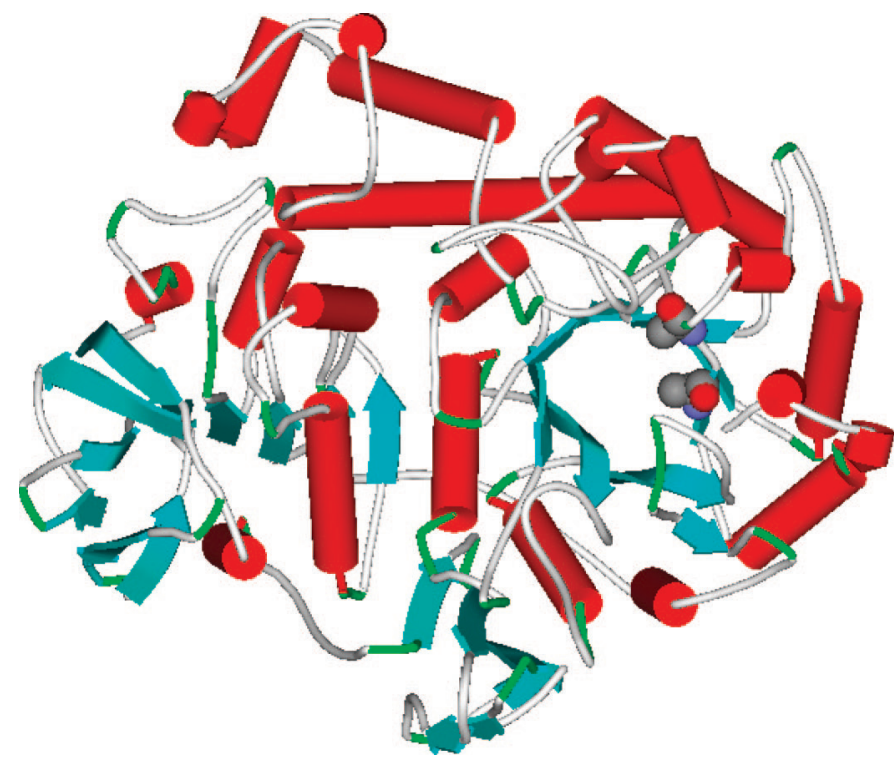

Figure 2. Geobacillus stearothermophilus $\beta$-galactosidase BgaB, with the secondary structures shown in cartoon representation and labeled according to BgaB nomenclature. $\alpha$-Helices are shown as cylinders (red), $\beta$-strands are shown as flat arrows (blue), loops are shown in gray, and Ile42 and Ser39 residues are shown in space-filling format. Color version available online.

which is common to the glycoside hydrolase family. The TIM barrel contains 379 AA residues (residues 4-382), and an inner core of 8 parallel $\beta$-strands, which are surrounded by $8 \alpha$-helices. Residue 42 is in the $\mathrm{C}$-terminal loop between $\beta-2$ and $\alpha-2$, and residue 39 is at the C-terminal end of $\beta-2$, which constitutes part of the TIM barrel (Figure 2). It has been reported that, in the process of reverse engineering of this fold, $\beta$-central residues, $\beta$-strand stops, and inter-subunit salt-bridges are important for protein structure and function, whereas $\alpha$-helix capping, $\alpha$-stops, turn sequences, and residues at the interface of $\alpha$-helices and $\beta$-strands are highly mutable (Bhardwaj, 2014). Thus, the AA substitutions at position 42 might have affected enzyme activity and thermostability by influencing the flexibility of the $\alpha-2$ helix containing the interacting residues Glu303 and Ser39. It is now generally recognized that some residues in the catalytic cleft of glycoside hydrolases, other than the catalytic residues, might play an essential role in catalytic activity. Such residues can be involved not only in enzyme-substrate interactions or structure stabilization, but also in the creation of interaction networks essential for catalysis (Lacombe-Harvey et al., 2013). As a previous study has indicated that the stabilizing AA substitutions are positioned in loops on the periphery of the active domain TIM barrel (Höcker, 2005), we suggest that Ile42 is influencing the catalytic nucleophile residue and the thermostability of the enzyme by optimizing structural interactions.

\section{Functional Diversity at Position 42}

Our data suggest that Ile42 could be a functional diversity position in the enzyme mechanism. To address this issue, we aligned the sequences containing the residues equivalent to Ile42 in the $\beta$-galactosidases from the GH42 family and found 4 variants: Glu, Val, Thr, and Met in this position (Figure 3). In our case, substitution of Ile42 with Glu resulted in a decrease in specific activity and an increase in thermal stability (Table 2). This loss of activity can be partly explained by the drastic decrease in the $k_{\text {cat }}$ values observed for the Glu mutant (Table 3). In addition, the increased $K_{m}$ value for the Glu mutant indicated an impaired substrate binding (Table 3). Although substitution of Ile42 with Thr or Met had only a slight effect on enzyme activity, these mutations resulted in an increase in thermal stability. It is curious that replacement of Ile42 with Val did not cause a dramatic loss of activity and thermal stability. Considering the above discussion, it is quite plausible that the mutation of Ile42 to polar AA, with their ability to form hydrogen bonds, is the consequence of evolutionary pressure leading to an increase in thermal stability.

\section{CONCLUSIONS}

In general, residues other than the catalytic AA play essential roles in maintaining the integrity of enzymatic function, and glycoside hydrolases are no exception. In thermostable $\beta$-galactosidase $\mathrm{BgaB}$, some key residues have been identified and are believed to play roles in modulating the substrate affinity of catalytic residues, hydrolyzing glycosidic bonds, stabilizing the protein structure, and participating in substrate-enzyme interactions. In the past few years, several studies have shown that, apart from the carboxylic catalytic residues, other residues are crucial for catalysis. In this study, we demonstrated that residue Ile42, differentially conserved in the GH42 family, substantially contributes to the enzymatic function of the $\beta$-galactosidase $\mathrm{BgaB}$ from G. stearothermophilus. We showed that Ile42 could play a dual role by influencing not only the formation of hydrogen bonds but also by modulating substrate binding. Moreover, our data suggest that Ile42 could be a functional diversity position in the enzyme mechanism, which influences the nucleophilic residue in the catalytic triad as well as the thermostability of the enzyme by optimizing hydrogen bonding between the residues 


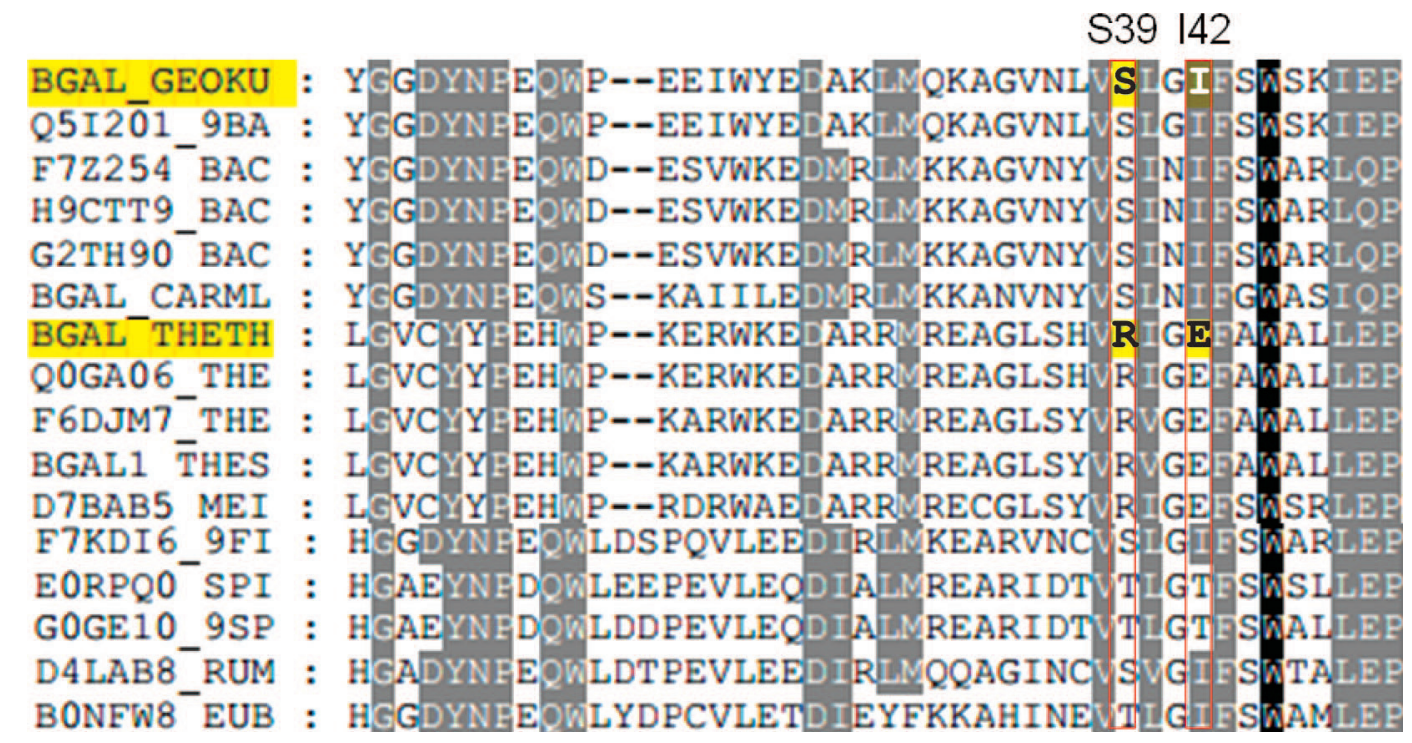

Figure 3. Comparison of residues equivalent to Ser39 and Ile42 in $\beta$-galactosidases BgaB (BGAL_GEOKU) and A4- $\beta$-Gal (BGAL_THETH) from glycoside hydrolase family 42 (GH42). Residues in black are highly conserved; other partially conserved regions are depicted in grey. Residues Ser39 (S39) and Ile42 (I42) of BgaB, and the equivalent positions in A4- $\beta$-Gal are shown in bold font (yellow highlighting). Color version available online.

equivalent to Ile42 and Ser39 in the $\beta$-galactosidases from GH42.

\section{ACKNOWLEDGMENTS}

This work was supported financially by the National Natural Science Foundation of China (31301523, 31171636, 30901129), the National High Technology Research and Development Program of China (2011AA100905), the Program for New Century Excellent Talents (NCET-13-0831), the Program for Changjiang Scholars and Innovative Research Team in University (IRT1249), Anhui Provincial University Natural Science Research Project (KJ2013B187), and Anhui Provincial Chuzhou University Research Startup Foundation (2012qd14).

\section{REFERENCES}

Arnold, F. H., and G. Georgiou. 2003. Directed Evolution Library Creation: Methods and Protocols. Methods in Molecular Biology Series, Vol. 231. Humana/Springer, Totowa, NJ.

Bhardwaj, A. 2014. Investigating the role of site-specific synonymous variation in disease association studies. Mitochondrion 16:83-88.

Bhattacharya, A., Z. Wunderlich, D. Monleon, R. Tejero, and G. T. Montelione. 2008. Assessing model accuracy using the homology modeling automatically software. Proteins 70:105-118.

Bradford, M. M. 1976. A rapid and sensitive method for the quantitation of microgram quantities of protein utilizing the principle of protein-dye binding. Anal. Biochem. 72:248-254.

Chen, W., H. Chen, Y. Xia, J. Zhao, F. Tian, and H. Zhang. 2008. Production, purification, and characterization of a potential thermostable galactosidase for milk lactose hydrolysis from Bacillus stearothermophilus. J. Dairy Sci. 91:1751-1758.
Daga, P. R., R. Y. Patel, and R. J. Doerksen. 2010. Template-based protein modeling: Recent methodological advances. Curr. Top. Med. Chem. 10:84-94.

Daudé, D., C. M. Topham, M. Remaud-Simeon, and I. Andre. 2013. Probing impact of active site residue mutations on stability and activity of Neisseria polysaccharea amylosucrase. Protein Sci. 22:1754-1765.

Desai, B. J., Y. Goto, A. Cembran, A. A. Fedorov, S. C. Almo, J. Gao, H. Suga, and J. A. Gerlt. 2014. Investigating the role of a backbone to substrate hydrogen bond in OMP decarboxylase using a site-specific amide to ester substitution. Proc. Natl. Acad. Sci. USA 111:15066-15071.

Dong, Y. N., X. M. Liu, H. Q. Chen, Y. Xia, H. P. Zhang, H. Zhang, and W. Chen. 2011. Enhancement of the hydrolysis activity of beta-galactosidase from Geobacillus stearothermophilus by saturation mutagenesis. J. Dairy Sci. 94:1176-1184.

Duarte, P. M., M. d. P. F. G. Torres, J. A. Teixeira, and L. R. Rodrigues. 2010. Galacto-oligosaccharides: Production, properties, applications, and significance as prebiotics. Comp. Rev. Food Sci. Food Safety 9:438-454.

Ghollasi, M., M. Ghanbari-Safari, and K. Khajeh. 2013. Improvement of thermal stability of a mutagenised alpha-amylase by manipulation of the calcium-binding site. Enzyme Microb. Technol. 53:406-413.

Gupta, R., T. Govil, N. Capalash, and P. Sharma. 2012. Characterization of a glycoside hydrolase family 1 beta-galactosidase from hot spring metagenome with transglycosylation activity. Appl. Biochem. Biotechnol. 168:1681-1693.

Hangasky, J. A., G. T. Ivison, and M. J. Knapp. 2014. Substrate positioning by Gln (239) stimulates turnover in factor inhibiting HIF, an alphaKG-dependent hydroxylase. Biochemistry 53:5750-5758.

Hidaka, M., S. Fushinobu, N. Ohtsu, H. Motoshima, H. Matsuzawa, H. Shoun, and T. Wakagi. 2002. Trimeric crystal structure of the glycoside hydrolase family 42 beta-galactosidase from Thermus thermophilus A4 and the structure of its complex with galactose. J. Mol. Biol. 322:79-91.

Höcker, B. 2005. Directed evolution of (beta/alpha) $)_{(8)}$-barrel enzymes. Biomol. Eng. 22:31-38.

Ji, E. S., N. H. Park, and D. K. Oh. 2005. Galacto-oligosaccharide production by a thermostable recombinant $\beta$-galactosidase from Thermotoga maritime. World J. Microbiol. Biotechnol. 21:759-764. 
Lacombe-Harvey, M. È., M. Fortin, T. Ohnuma, T. Fukamizo, T. Letzel, and R. Brzezinski. 2013. A highly conserved arginine residue of the chitosanase from Streptomyces sp. N174 is involved both in catalysis and substrate binding. BMC Biochem. 14:23.

Marin-Navarro, J., D. Talens-Perales, A. Oude-Vrielink, F. J. Canada, and J. Polaina. 2014. Immobilization of thermostable beta-galactosidase on epoxy support and its use for lactose hydrolysis and galactooligosaccharides biosynthesis. World J. Microbiol. Biotechnol. 30:989-998.

Mashiach, E., D. Schneidman-Duhovny, A. Peri, Y. Shavit, R. Nussinov, and H. J. Wolfson. 2010. An integrated suite of fast docking algorithms. Proteins 78:3197-3204.

McLachlan, M. J., T. W. Johannes, and H. Zhao. 2008. Further improvement of phosphite dehydrogenase thermostability by saturation mutagenesis. Biotechnol. Bioeng. 99:268-274.

McNicholas, S., E. Potterton, K. S. Wilson, and M. E. Noble. 2011. Presenting your structures: The CCP4mg molecular-graphics software. Acta Crystallogr. D Biol. Crystallogr. 67:386-394.

Oliveira, C., P. M. Guimaraes, and L. Domingues. 2011. Recombinant microbial systems for improved beta-galactosidase production and biotechnological applications. Biotechnol. Adv. 29:600-609.

Park, A. R., H. J. Kim, J. K. Lee, and D. K. Oh. 2010. Hydrolysis and transglycosylation activity of a thermostable recombinant beta-glycosidase from Sulfolobus acidocaldarius. Appl. Biochem. Biotechnol. 160:2236-2247.

Petzelbauer, I., B. Nidetzky, D. Haltrich, and K. D. Kulbe. 1999. Development of an ultra-high-temperature process for the enzymatic hydrolysis of lactose. I. The properties of two thermostable betaglycosidases. Biotechnol. Bioeng. 64:322-332.

Placier, G., H. Watzlawick, C. Rabiller, and R. Mattes. 2009. Evolved beta-galactosidases from Geobacillus stearothermophilus with improved transgalactosylation yield for galacto-oligosaccharide production. Appl. Environ. Microbiol. 75:6312-6321.
Sali, A., L. Potterton, F. Yuan, H. van Vlijmen, and M. Karplus. 1995. Evaluation of comparative protein modeling by MODELLER. Proteins 23:318-326.

Schmidt, M., and P. Stougaard. 2010. Identification, cloning and expression of a cold-active beta-galactosidase from a novel Arctic bacterium, Alkalilactibacillus ikkense. Environ. Technol. 31:11071114 .

Schneidman-Duhovny, D., Y. Inbar, R. Nussinov, and H. J. Wolfson. 2005. PatchDock and SymmDock: Servers for rigid and symmetric docking. Nucleic Acids Res. 33(Web Server issue):W363-367.

Surzhik, M. A., A. E. Shmidt, E. A. Glazunov, D. L. Firsov, and M. G. Petukhov. 2014. (Introduction of additional thiol groups into glucoamylase in Aspergillus awamori and their effect on the thermal stability and catalytic activity of the enzyme). Prikl. Biokhim. Mikrobiol. 50:139-146. [Article in Russian]

Tiwari, M. K., V. C. Kalia, Y. C. Kang, and J. K. Lee. 2014. Role of a remote leucine residue in the catalytic function of polyol dehydrogenase. Mol. Biosyst. 10:3255-3263.

Torres, S., J. E. Sayago, R. M. Ordonez, and M. I. Isla. 2010. A colorimetric method to quantify endo-polygalacturonase activity. Enzyme Microb. Technol. 48:123-128.

Viborg, A. H., T. Katayama, M. Abou Hachem, M. C. Andersen, M. Nishimoto, M. H. Clausen, T. Urashima, B. Svensson, and M. Kitaoka. 2014. Distinct substrate specificities of three glycoside hydrolase family 42 beta-galactosidases from Bifidobacterium longum ssp. infantis ATCC 15697. Glycobiology 24:208-216.

Wallace, A. C., R. A. Laskowski, and J. M. Thornton. 1995. LIGPLOT: A program to generate schematic diagrams of proteinligand interactions. Protein Eng. 8:127-134.

Xia, Y., J. Zhao, H. Chen, X. Liu, Y. Wang, F. Tian, H. P. Zhang, H. Zhang, and W. Chen. 2010. Extracellular secretion in Bacillus subtilis of a cytoplasmic thermostable beta-galactosidase from Geobacillus stearothermophilus. J. Dairy Sci. 93:2838-2845. 